\title{
Merging fluxgate and induction coil data to produce low-noise geomagnetic observatory data meeting the INTERMAGNET definitive $1 \mathrm{~s}$ data standard
}

\author{
Heinz-Peter Brunke ${ }^{1}$, Rudolf Widmer-Schnidrig ${ }^{2}$, and Monika Korte ${ }^{1}$ \\ ${ }^{1}$ GFZ German Research Centre for Geosciences, Telegrafenberg, 14473 Potsdam, Germany \\ ${ }^{2}$ Black Forest Observatory, Heubach 207, 77709 Wolfach, Germany \\ Correspondence to: Heinz-Peter Brunke (brunke@gfz-potsdam.de) \\ Received: 3 March 2017 - Discussion started: 15 May 2017 \\ Revised: 8 August 2017 - Accepted: 21 August 2017 - Published: 9 November 2017
}

\begin{abstract}
For frequencies above $30 \mathrm{mHz}$ the instrument intrinsic noise level of typical fluxgate magnetometers used at geomagnetic observatories usually masks ambient magnetic field variations on magnetically quiet days. This is especially true for stations located at middle and low latitudes, where variations are generally smaller than at high latitudes. INTERMAGNET has set a minimum quality standard for definitive $1 \mathrm{~s}$ data. Natural field variations referred to as pulsations (Pc-1, Pc-2, Pi-1) fall in this band. Usually their intensity is so small that they rarely surpass the instrumental noise of fluxgate magnetometers. Moreover, highquality magnetic field observations in the band $30 \mathrm{mHz}-$ $0.5 \mathrm{~Hz}$ contain interesting information, e.g., for the study of ionospheric electron interactions with electromagnetic ion cyclotron plasma waves.

We propose a method to improve $1 \mathrm{~Hz}$ observatory data by merging data from the proven and tested fluxgate magnetometers currently in use with induction coil magnetometers into a single data stream. We show how measurements of both instruments can be combined without information loss or phase distortion.

The result is a time series of the magnetic field vector components, combining the benefits of both instruments: longterm stability (fluxgate) and low noise at high frequencies (induction coil). This new data stream fits perfectly into the data management procedures of INTERMAGNET and meets the requirements defined in the definitive $1 \mathrm{~s}$ data standard. We describe the applied algorithm and validate the result by comparing power spectra of the fluxgate magnetometer output with the merged signal. Daily spectrograms from the Niemegk observatory show that the resulting data series re-
\end{abstract}

veal information at frequencies above $30 \mathrm{mHz}$ that cannot be seen in raw fluxgate data.

\section{Introduction}

Conventional fluxgate magnetometers used at geomagnetic observatories are optimized towards long-term stability as their main purpose. Even though they have excellent properties to measure the low-frequency part of the magnetic spectrum, their noise usually surpasses the natural background field variations at frequencies higher than $30 \mathrm{mHz}$ at quiet days and mid- or low-latitude observatories. A commonly used instrument is the FGE fluxgate magnetometer (Pedersen and Merenyi, 2016). However, the demand for low-noise, high-frequency observatory data is increasing. Variations in the band $30 \mathrm{mHz}-0.5 \mathrm{~Hz}$ correspond to plasma waves, which are important for radiation belt physics. Electrons interacting with such waves can be scattered into the loss cone and reduce the energy content of the radiation belt. Pc-1 pulsations ( $f>0.1 \mathrm{~Hz}$ ) can be caused by electromagnetic ion cyclotron (EMIC) waves, which is the subject of many recent investigations (e.g., Shprits et al., 2016 or Usanova et al., 2014).

A quality standard for observatory data has been defined in technical note TN6 of 2 October 2014 INTERMAGNET definitive $1 \mathrm{~s}$ data standard (Turbitt, 2014). This INTERMAGNET standard requires a noise level less than $10 \mathrm{pT} / \sqrt{\mathrm{Hz}}$ at $0.1 \mathrm{~Hz}$. A specification that can hardly be met by fluxgate magnetometers currently used in magnetic observatories. Even though fluxgate magnetometers with low 
noise level at high frequencies have been presented (e.g., Korepanov, 2007), it is not desirable to replace approved observatory magnetometers that have proven long-term stability, as long-term absolute stability is the main requirement for geomagnetic observatories. Moreover, it is not desirable to discontinue a long-standing data series.

In the frequency band $30 \mathrm{mHz}-0.5 \mathrm{~Hz}$, natural magnetic signals are relatively rare and usually of small intensity. Hence, it is often termed the "dead band" in magnetotellurics. Such signals disappear in the noise because of their small intensity. In order to overcome this "blind spot" of magnetometers currently in use, we propose to improve the quality of observatory data by merging fluxgate data with induction coil data. At high frequencies induction coils have very little noise but provide no long-term stability. Here we present a method to numerically merge data of both instruments. The result is a single time series combining the benefits of both instruments: long-term stability (fluxgate) and low noise at high frequencies (induction coil). The result can be understood as $1 \mathrm{~s}$ fluxgate data, noise filtered without loss of information on phase or amplitude. The resulting data product fits perfectly in the INTERMAGNET data processing scheme established at magnetic observatories. It exceeds the INTERMAGNET noise requirement of $10 \mathrm{pT} / \sqrt{\mathrm{Hz}}$ at $0.1 \mathrm{~Hz}$ by far. As a consequence, three decimal digits of the merged data are valid $(1 \mathrm{pT})$. This is one digit more than required in the INTERMAGNET definitive $1 \mathrm{~s}$ data standard. The third digit is needed to describe weak magnetic signals that can now be observed. Otherwise the effect of quantization noise can be seen in the spectrum as shown in Fig. 3 .

\section{Noise considerations}

As shown in Figs. 1 and 3, the instrument-inherent noise of a fluxgate magnetometer can be identified in the data as white noise for frequencies above $30 \mathrm{mHz}$. On a normal day the part of the natural signal with frequencies above $30 \mathrm{mHz}$ vanishes in the noise. Korepanov (2007) shows a typical power spectral density (PSD) of the natural field at the Dourbes magnetic observatory and compares it to noise of their new magnetometer kept in a shielding box (Fig. 1). We added a green line for the spectral noise density of the FGE in the frequency band $30 \mathrm{mHz}-0.5 \mathrm{~Hz}$, as observed at the Niemegk observatory (NGK) and shown in Fig. 3. We marked the INTERMAGNET requirement of $10 / \sqrt{\mathrm{Hz}}$ at $0.1 \mathrm{~Hz}$ as a blue dot and added the noise of an induction coil magnetometer (MFS05, METRONIX) after Ritter (2001) as a red line. Whereas the fluxgate spectral noise density remains constant at high frequencies, the spectral noise density of an induction coil decreases towards higher frequencies by $20 \mathrm{~dB}$ (factor 10) per decade up to a frequency of $100 \mathrm{~Hz}$ (Ritter, 2001). At $1 \mathrm{~Hz}$ its spectral noise density is at least 2 orders of magnitudes lower than the noise of any fluxgate magnetometer.

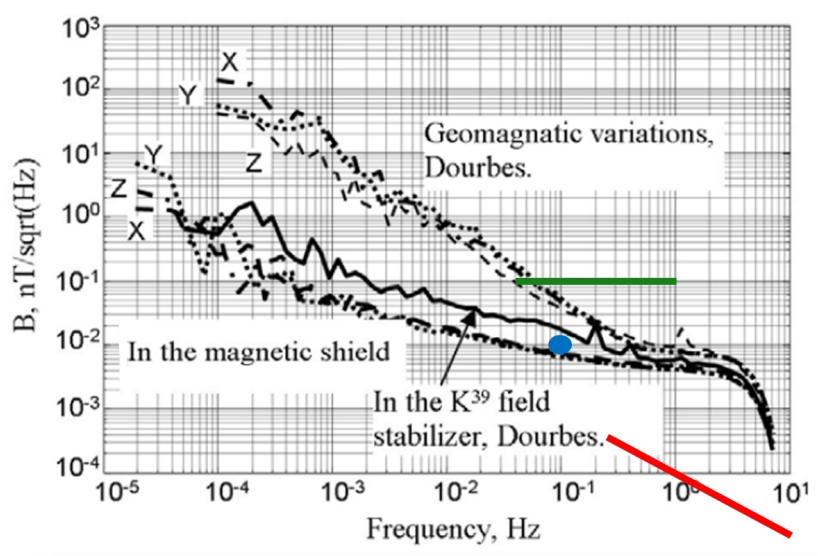

Figure 1. Noise spectra of a magnetometer prototype and of measured natural geomagnetic variations (modified after Korepanov, 2007). We added the high-frequency noise level of the FGE magnetometer as measured at NGK (green line) and the high-frequency noise of a typical induction coil magnetometer (red line). The blue dot shows the specification required by the INTERMAGNET definitive $1 \mathrm{~s}$ data standard (Turbitt, 2014).

Instrument noise at very long periods from days to years is equivalent to instrument long-term stability. This is the most important quality feature of an observatory fluxgate magnetometer. As described in the following, merging data of both instruments does not in any way effect the long-term stability.

\section{Combining fluxgate and induction coil magnetometer}

We take advantage of the fact that the signal of an induction coil magnetometer is proportional to the time derivative of the magnetic field component along the axis of the coil. In the following we consider exemplary the $X$ component. For each sample time $t_{0}$ we look at values $X_{\mathrm{FG}}\left(t_{i}\right)$ registered by the fluxgate magnetometer at times $t_{i}$ varying from $t_{0}-T_{\mathrm{S}} N$ to $t_{0}+T_{\mathrm{S}} N$, with the sample width $T_{\mathrm{s}}$. Additionally we need recordings of the induction coil at time $t_{i}$. As result of our algorithm the value $B_{X}\left(t_{0}\right)$ is a more precise value of the magnetic field at time $t_{0}$. The method works comparable to a filter. It acts on the measurements at all times $t_{i}$ varying from $t_{0}-T_{\mathrm{S}} N$ to $t_{0}+T_{\mathrm{s}} N$ and produces one output value for time $t_{0}$. For each new $t_{0}$ the entire process has to be repeated using a new set of $2 N+1$ measurements. Even though quite a number of measurements are involved, the numerical effort is restricted to just solving the $3 \times 3$ matrix of Eq. (5).

Integrating induction coil data provides a curve which is comparable to the fluxgate data. The integration constant $B_{X}\left(t_{0}\right)$ is the field at the start time $t_{0}$ of the integration. Due to the low noise content of an induction coil, this curve (blue line in Fig. 2) is smooth compared to fluxgate data. Our approach is to fit the former to the fluxgate data (Fig. 2b). Fit- 

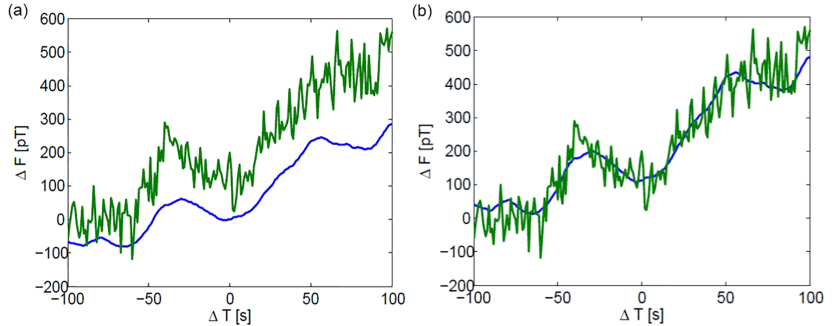

Figure 2. (a) Fluxgate data (green) and integral of induction coil signal (blue). (b) Blue curve fitted to FG data by adapting $C, \Delta U$ and $B_{X}\left(t_{0}\right)$ in Eq. (2). In this plot, fluxgate data $\Delta F$ are referred to an arbitrary base value to get handy numerical values on the axis of ordinate.

ting a smooth curve effectively eliminates instrument noise of the fluxgate by averaging. However, the shape of the fitted curve results from the induction coil measurements and the loss of information due to averaging is replaced by information stemming from the induction coil.

For given time $t_{0}$ and a variable time $t_{i}$ in its environment, data of fluxgate $X_{\mathrm{FG}}$ and induction coil magnetometer $\dot{X}_{\mathrm{IC}}$ can be expected to satisfy the following equation, assuming that both sensors are aligned with the $x$ axis:

$X_{\mathrm{FG}}\left(t_{i}\right)-B_{X}\left(t_{0}\right)=\int_{t_{0}}^{t_{i}} \dot{X}_{\mathrm{IC}}(t) \mathrm{d} t$.

Assuming that the induced voltage measured over the induction coil is object to scale factor $C$ and offset $\Delta U$,

$X_{\mathrm{FG}}\left(t_{i}\right)-B_{X}\left(t_{0}\right)=\int_{t_{0}}^{t_{i}}\left(C \cdot U_{\mathrm{IC}}(t)+\Delta U\right) \mathrm{d} t$.

The blue line in Fig. 2a is achieved by evaluating the integral from the fixed central time $t_{0}$ to a time $t_{i}$, with $i$ varying from $-N$ to $+N$ and applied to an actually measured induction coil data set. The corresponding time series of a fluxgate magnetometer is given as green line.

The blue line (induction coil) is much smoother than the green one (fluxgate data). It can be fitted to the fluxgate data by choosing the right values for the parameters $\Delta U, C$ and $B_{X}\left(t_{0}\right)$, as shown in the right panel of Fig. 2. For this fit, Eq. (2) has to be solved for $\Delta U, C$ and $B_{X}\left(t_{0}\right)$ as unknowns. The effect of $B_{X}\left(t_{0}\right)$ shifts the blue curve vertically, $\Delta U$ tilts it and $C$ changes the amplitudes. With fixed $t_{0}$ and varying $t_{i}$, Eq. (2) delivers one equation for each $t_{i}$ to determine these unknowns. As shown in Fig. 2 we use a time interval of $200 \mathrm{~s}$. The time spread of $200 \mathrm{~s}$ was determined empirically. A longer time interval increases the number of equations and therefore might improve the numerical accuracy of the result. However, the time interval must be small enough to ensure not to distort the fluxgate signal at lower frequencies. In Sect. 4 we show that a further improvement is not necessary because the "actual natural" signal is clearly resolved up to the Nyquist frequency of $0.5 \mathrm{~Hz}$.

Assuming that both data series are available at a sample rate of $1 \mathrm{~Hz}$ and approximating the integral by a sum, Eq. (2) can be written in the following discrete form:

$\sum_{t_{0}}^{t_{i}}\left(C \cdot U_{\mathrm{IC}}(t)+\Delta U\right) T_{\mathrm{s}}-B_{X}\left(t_{0}\right)=X_{\mathrm{FG}}\left(t_{i}\right)$.

Assuming that $T_{\mathrm{S}}=1 \mathrm{~s}$, one equation is derived for each sample time $t_{i}$. If $i$ varies from $-N$ to $+N$, we get the following conditional system for the parameters $C, \Delta U$ and $B_{X}\left(t_{0}\right)$. The system of equations is overdetermined and we have to introduce small residuals $r_{i}$ :

$$
\begin{aligned}
& C \cdot S_{N}+\Delta U \cdot N+B_{X}\left(t_{0}\right)=X_{\mathrm{FG}}\left(t_{N}\right)+r_{N} \\
& \cdots \\
& C \cdot S_{i}+\Delta U \cdot i+B_{X}\left(t_{0}\right)=X_{\mathrm{FG}}\left(t_{i}\right)+r_{i} \\
& \cdots \\
& C \cdot S_{-N}+\Delta U \cdot(-N)+B_{X}\left(t_{0}\right)=X_{\mathrm{FG}}\left(t_{-N}\right)+r_{-N} .
\end{aligned}
$$

This is a system of linear equations of the form $G \cdot x=y+$ $r$. With $S_{i}=T_{\mathrm{s}} \sum_{k=0}^{i-1} U_{\mathrm{IC}}(k)$ and written as explicit matrices,

$$
\begin{gathered}
\left(\begin{array}{ccc}
S_{N} & N & 1 \\
& \vdots & \\
S_{i} & i & 1 \\
& \vdots & \\
S_{-N} & -N & 1
\end{array}\right) \cdot\left(\begin{array}{c}
C \\
\Delta U \\
B_{X}\left(t_{0}\right)
\end{array}\right) \\
=\left(\begin{array}{c}
X_{\mathrm{FG}}\left(t_{N}\right) \\
\vdots \\
X_{\mathrm{FG}}\left(t_{i}\right) \\
\vdots \\
X_{\mathrm{FG}}\left(t_{-N}\right)
\end{array}\right)+\left(\begin{array}{c}
r_{N} \\
\vdots \\
r_{i} \\
\vdots \\
r_{-N}
\end{array}\right)
\end{gathered}
$$

Setting $N$ to 100 , we get a time interval of $200 \mathrm{~s}$, leading to 201 equations. Obviously we have more than three conditional equations at hand and a solution must be found in the sense of minimizing the mean square of the residuals. The so-called normal equations (Schmucker and Weidelt, 1975) are $x=\left(G^{T} \cdot G\right)^{-1} \cdot\left(G^{T} \cdot y\right)$. More explicit for this case, 


$$
\begin{aligned}
\left(\begin{array}{c}
C \\
\Delta U \\
B_{X}\left(t_{0}\right)
\end{array}\right)=\left(\begin{array}{ccc}
\sum_{i=-N}^{N} S_{i}^{2} & \sum_{i=-N}^{N} i \cdot S_{i} & \sum_{i=-N}^{N} S_{i} \\
\sum_{i=-N}^{N} i \cdot S_{i} & N(N+1)(2 N+1) / 3 & 0 \\
\sum_{i=-N}^{N} S_{i} & 0 & 2 N+1
\end{array}\right) \\
\\
\qquad\left(\begin{array}{c}
\sum_{i=-N}^{N} S_{i} X_{\mathrm{FG}}\left(t_{i}\right) \\
\sum_{i=-N}^{N} i X_{\mathrm{FG}}\left(t_{i}\right) \\
\sum_{i=-N}^{N} X_{\mathrm{FG}}\left(t_{i}\right)
\end{array}\right)
\end{aligned}
$$

As a result we get $B_{X}\left(t_{0}\right)$, the improved field value at the time $t_{0}$. Note that the numeric effort for this calculation is very small. It is restricted to the inversion of a $3 \times 3$ matrix and calculating six sums in Eq. (5) for each sample of the resulting time series. The sums do not even have to be evaluated for each new $t_{0}$. They can be updated from the previous time step.

\section{Results}

The success of merging both instruments can best be demonstrated by comparing power spectra of unprocessed FGE and merged data and looking at spectrograms of the respective data series. The induction coil used is a proprietary development at the NGK. In Fig. 3 PSDs of the fluxgate data as measured in NGK are compared to PSD of merged data. The instrument-inherent noise can clearly be identified in the unprocessed data as the white noise (constant noise level) of $0.01 \mathrm{nT}^{2} / \mathrm{Hz}$ for frequencies above $40 \mathrm{mHz}$. At the same frequencies the PSDs of the merged data show a continuing linear decay as expected for the natural field. Very little influence of noise is visible in the PSD of the merged data. At frequencies lower than $10 \mathrm{mHz}$, the spectra of FGE and merged data are identical.

As shown in Fig. 4 the coherence between merged data and FGE data is perfect at low frequencies $(<0.01 \mathrm{~Hz})$. At high frequencies $(>0.03 \mathrm{~Hz}$ ) the coherence is very good between merged data and induction coil data. It is not perfect due to the low energy content of the signal at these frequencies. A very high coherence is also observed between FGE and induction coil data at frequencies between 0.003 and $0.03 \mathrm{~Hz}$. In this frequency band both instruments are most comparable, allowing us to check alignment and intercalibration of both instruments. Induction coils are usually run at a GPS synchronized data logger. This allows us to verify the correct time stamping of the FGE data logger as proposed in the INTERMAGNET definitive $1 \mathrm{~s}$ data standard (Turbitt, 2014). For such a test a natural or artificial signal clearly surpassing the FGE white noise level is required.
Figure 5 shows spectrograms for 3 selected days. The spectrograms are overlain with time series of the field (black line). Panels (a) and (c) are directly comparable as both cover the 2 February 2016. Panel (a) is based on merged data and panel (c) on fluxgate data only. Whereas in panel (a) the spectral content is clearly resolved up to the Nyquist frequency of $0.5 \mathrm{~Hz}$ in panel (c) only the strongest activity surpass the noise level in the frequency band from $30 \mathrm{mHz}$ up to $0.5 \mathrm{~Hz}$. These are possibly field line resonances and their lowering frequencies towards the nighttime is clearly visible in the merged data in panel (a). Panel (a) also shows with help of the overlain magnetogram that irregular pulsations (Pi-1 pulsation, no clear frequency) are related to the beginning of substorms. Panel (b) shows around 18:00 an example of a pulsation event with a clearly defined center frequency of about $300 \mathrm{mHz}$ (Pc-1 pulsation) and panel (d) shows an example of a pulsation whose main frequency rises from about $20 \mathrm{mHz}$ at $15: 30$ to $400 \mathrm{mHz}$ at $16: 30$. Additionally, panel (b) shows horizontal structures from 06:00 to 17:00 (16.2 s square wave) and sweeps with falling frequency starting at 06:30 and 09:00. Both are manmade contaminations. We found a lot of other remarkable signals revealed in such spectrograms, but discussing them is beyond the scope of this paper.

\section{Discussion}

Providing data to a user is generally linked to the commitment to the best possible accuracy. Speaking in terms of data series, this means to optimize the signal-to-noise ratio over the entire available bandwidth. Hence our effort improves magnetic data in view of the INTERMAGNET $1 \mathrm{~s}$ standard. We are well aware that merging two data sets does not produce new information. Merging data from different instruments at geomagnetic observatories is not a farfetched idea. On the contrary, it is already a standard technique when definitive data are produced by combining absolute observations from DI-theodolite and scalar magnetometer with vector variometer (fluxgate) data. But as INTERMAGNET does not cover induction coil data, applying our method makes additional information available to the INTERMAGNET community. Plots as presented in Fig. 5 could also be produced using both data sets separately. However, after applying our method the single $1 \mathrm{~s}$ data series as administered for many observatories worldwide by INTERMAGNET is sufficient. The effort of storing and exchange of data sampled at $1 \mathrm{~s}^{-1}$ as proposed by INTERMAGNET makes more sense if the entire possible bandwidth contains information about the Earth magnetic field. However, in order to take full advantage of the high-frequency information, the data we produce should be reported to at least three decimal digits (1 pT). Otherwise quantization noise becomes visible. The quantization noise level due to truncation is shown in Fig. 3. 


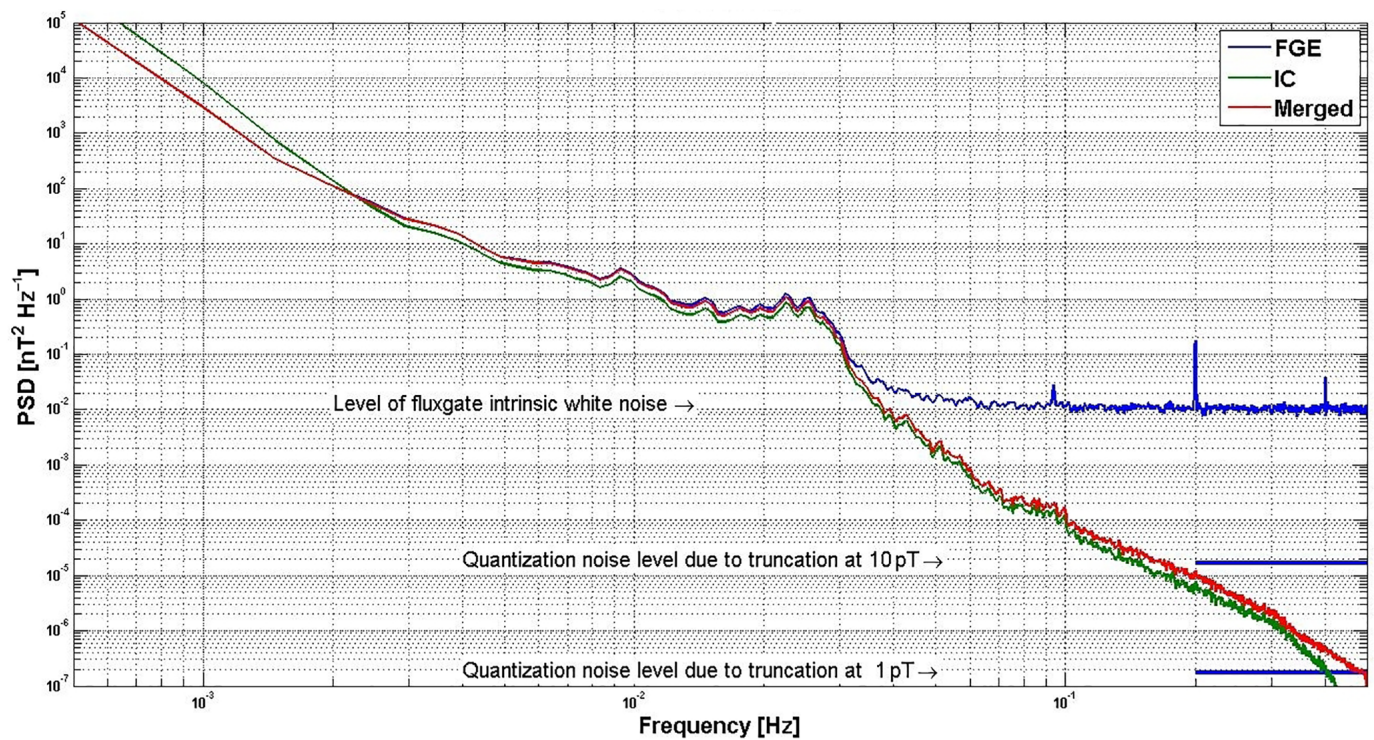

Figure 3. Spectra of unprocessed (FGE) and merged data of the entire day (17 July 2016). The level of the fluxgate-inherent white noise is clearly visible for frequencies over $0.04 \mathrm{~Hz}$ (blue), whereas the merged data show the continuing decay as expected for the natural field (red).

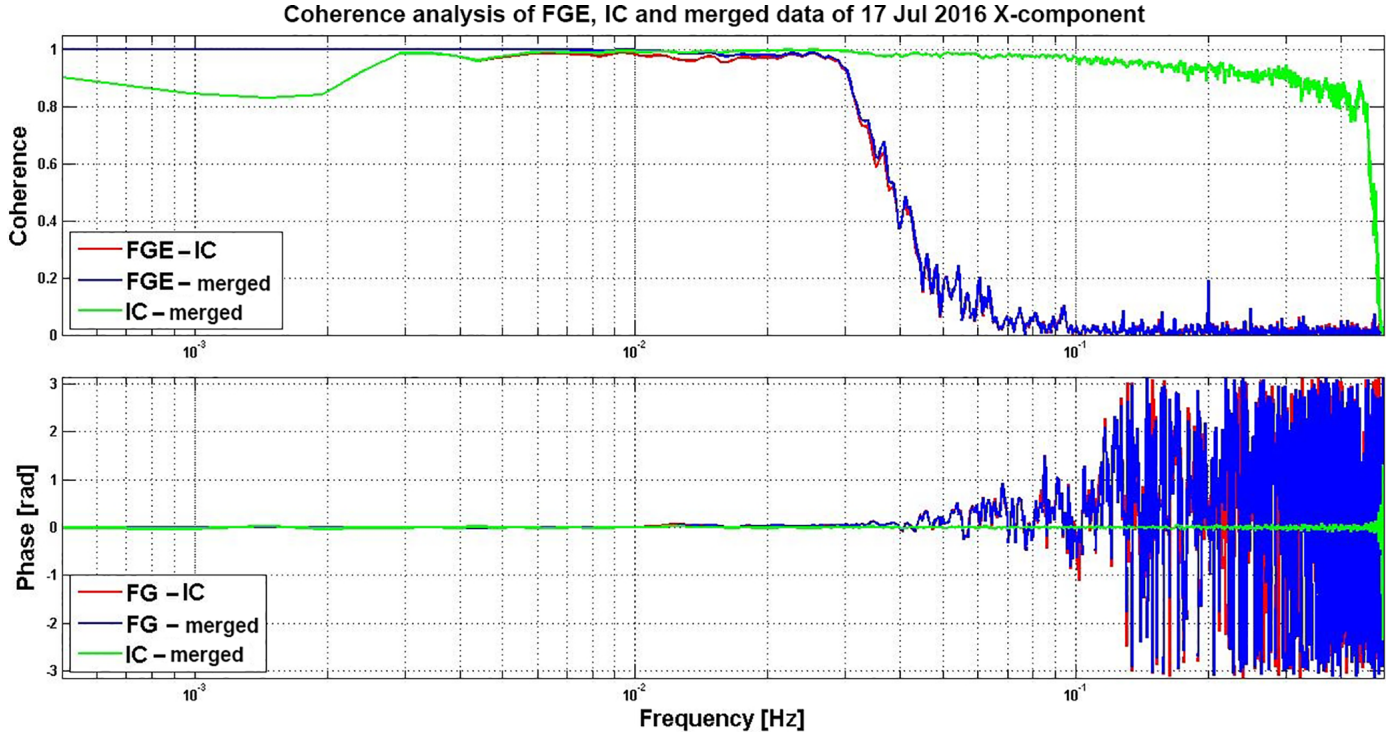

Figure 4. Coherences between FGE, merged data and induction coil (IC) data. A perfect coherence between merged data and FGE data (blue) is observed at low frequencies $(<0.01 \mathrm{~Hz})$. At high frequencies $(>0.03 \mathrm{~Hz})$ the coherence is high between merged data and IC data (green).

The INTERMAGNET $1 \mathrm{~s}$ standard prescribes a hardware low-pass filter with a corner frequency of $0.2 \mathrm{~Hz}$ for the fluxgate data. This filter has absolutely no effect to the result of the merging process. The high-frequency information stems from the induction coil side only. We verified this by numerical experiments.

The induction coil data are usually sampled at a rate higher than $1 \mathrm{~s}^{-1}$. These data must be down-sampled numerically to $1 \mathrm{~s}^{-1}$ prior to being used in our method. Variometer data are often explored in the time domain. The morphologies of events like sudden storm commencements, substorms or pulsations are investigated by visual or automated inspection. In merged data events with even very small amplitudes can be observed, as noise is eliminated to a large extent.

Our method can easily be extended to handle the high sample rate of induction coil data so that the usable signal bandwidth can be extended up to the $50 \mathrm{~Hz}$ power grid frequency - but that is beyond the scope of this paper.

We have to assume some preconditions for the application of our method: 

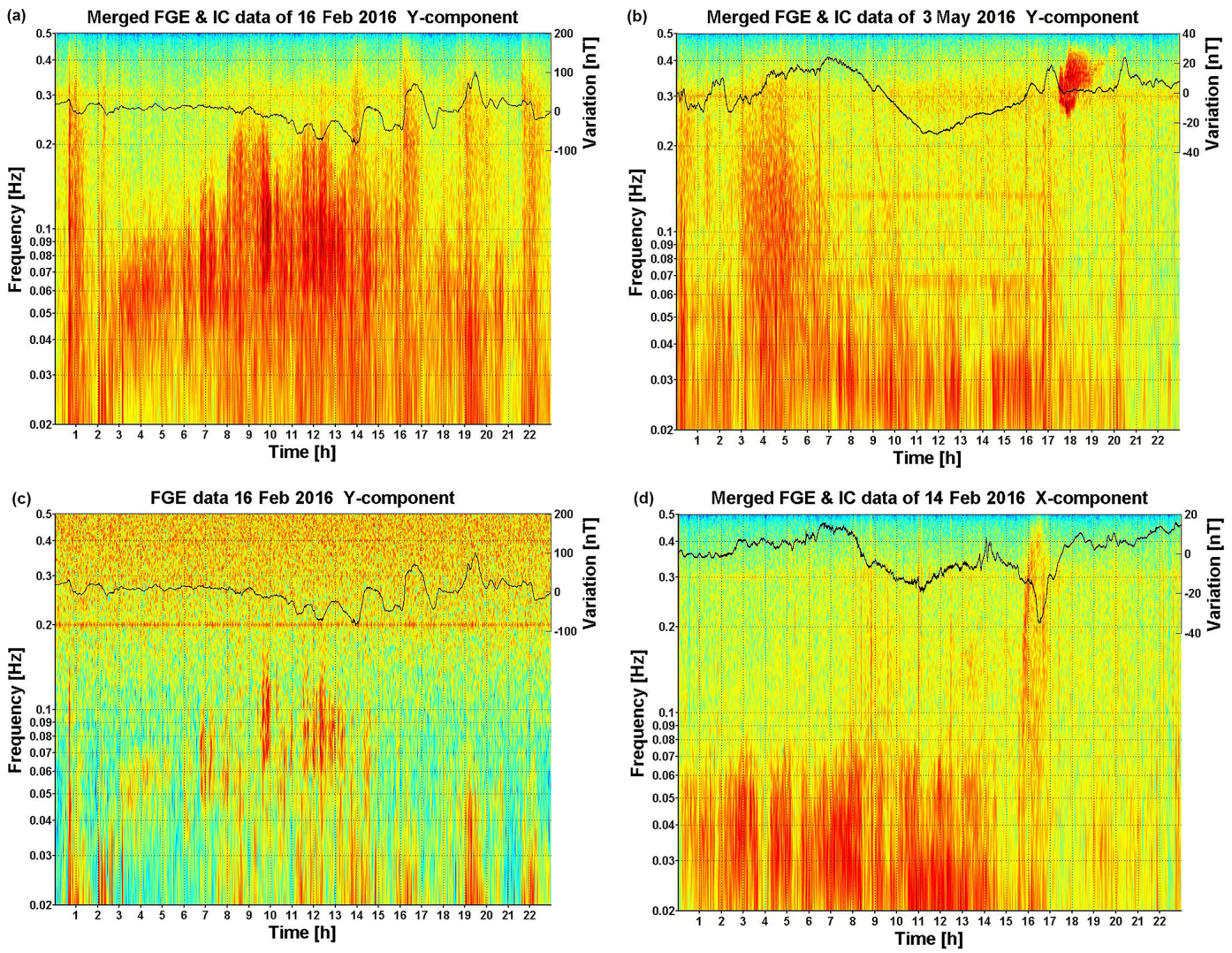

Figure 5. Spectrograms of 3 selected days. Panels (a) and (c) allow for a direct comparison between merged data (a) and fluxgate only (c). Interesting are the irregular pulsations related to substorm activity in panel (a), Pc-1 pulsations with clearly defined frequency of $300 \mathrm{mHz}$ in panel (b) at 18:00 and pulsation activity with rising frequency starting at 15:30 potentially related to EMIC waves in (d).

- We made the assumption that the sensors of the fluxgate and the induction coil magnetometer are perfectly aligned. If this is not the case, the induction coil signal will be affected by the other components. The fitting of both data will worsen. This could be accounted for by numerically rotating the data beforehand. For the determining the rotation matrix a larger data set is needed. The induction coils can be used down to relatively low frequencies, however, so that the overlap with the fluxgate is broad enough to determine the misalignment.

- We assume that the induction coil output is proportional to the time derivative of the magnetic field. Induction coils normally have a ferrite core and use electronic amplification (Ritter, 2001). Both can influence the linear transfer function in the frequency band from $30 \mathrm{mHz}$ to $1 \mathrm{~Hz}$. This effect could be compensated by a corresponding numeric amplitude pre-filtering. This point is especially important if the method should be extended to higher frequencies.

In the process of curve fitting depicted in Fig. 2, it is a good idea to weight points in the direct environment of the time of interest $\left(t_{0}\right)$ higher than more remote ones. We have

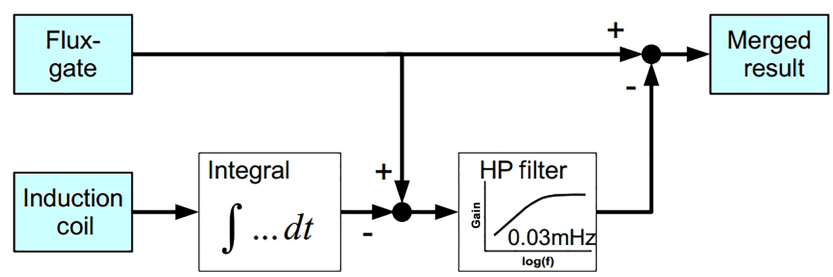

Figure 6. Data flow of a filtering method to merge fluxgate data and induction coil data. The cutoff frequency of the low-pass filter should be $30 \mathrm{mHz}$. Calibration of the induction coil data is not included.

had a good experience with a Gaussian filter. For the sake of simplicity we did not include weights in Eqs. (3) to (5).

The method we described above is inspired by the quite descriptive process of curve fitting in the time domain. Another way to do the merging is a filtering method. See Fig. 6 for a depiction of the data flow. The difference between fluxgate and integrated induction coil data is calculated and subsequently high-pass filtered. The obtained intermediate result contains the high-frequency content of the fluxgate data (mainly noise) and the inverse of the high-frequency signal content of the induction coil. In the intermediate result, drift 
of induction coil data and the integration constant is eliminated by the high-pass filter. In a final step the intermediate result is subtracted from the fluxgate data to get the merged output. The output contains the low-pass-filtered fluxgate data (the high-pass content has been subtracted) and the integrated, high-pass-filtered induction coil data. This means that the high-frequency content of the fluxgate, containing mainly noise, is eliminated in the resulting data and replaced by the corresponding high-frequency part of the induction coil side.

An advantage of the curve fitting method is that the scale factor of the induction coil is automatically corrected. The numerical effort of the curve fitting method may be slightly higher. Extending the curve fitting method to a higher sampling rate of the induction coils (e.g., up to $50 \mathrm{~Hz}$ ) is straightforward.

\section{Conclusions}

We have shown how data of fluxgate magnetometer and induction coil data can be merged. A numerical method based on the idea of fitting integrated induction coil data to fluxgate data has been presented in detail. The merged data set combines the excellent long-term stability of well-proven observatory fluxgate magnetometers with the low noise content of induction coil magnetometers at high frequencies. The resulting merged data differ from unprocessed fluxgate data only within the range of the intrinsic fluxgate noise $(<0.1 \mathrm{nT})$. Hence the long-term stability is in no way affected. We produce a data set that has the format of usual $1 \mathrm{~s}$ fluxgate data but surpasses the specification of the INTERMAGNET definitive $1 \mathrm{~s}$ data standard by far. The resulting data set reveals details like $\mathrm{Pc}-1, \mathrm{Pc}-2$ and $\mathrm{Pi}-1$ pulsation of even small intensities, which are not resolved by a fluxgate magnetometer. The spectral noise density in the resulting data set is up to the Nyquist frequency of $0.5 \mathrm{~Hz}$, clearly lower than the spectral density of the natural magnetic field even at days with low activity. Thus with our method we can fill the entire possible bandwidth resulting from the INTERMAGNET definitive $1 \mathrm{~s}$ data standard with meaningful information about the Earth magnetic field (Turbitt, 2014).

Code and data availability. The MATLAB source code implementing the method presented above is available by GFZ Data Services (Brunke et al., 2017). Test data sets are provided along with the source code. They have kindly been provided by the magnetic observatories Niemegk, Conrad and Eskdalemuir.

Competing interests. The authors declare that they have no conflict of interest.

Special issue statement. This article is part of the special issue "The Earth's magnetic field: measurements, data, and applications from ground observations (ANGEO/GI inter-journal SI)". It is a result of the XVIIth IAGA Workshop on Geomagnetic Observatory Instruments, Data Acquisition and Processing, Dourbes, Belgium, 4-10 September 2016.

Acknowledgements. We thank Sergey Khomutov, Lars Pedersen and one anonymous reviewer. We thank Kusumita Arora for editing. For the provision of test data we acknowledge Roman Leonhard, Ciaran Beggan and Jürgen Matzka. Kirsten Elger helped to publish the software with test data as data object. The article processing charges for this open-access publication were covered by a Research Centre of the Helmholtz Association.

The article processing charges for this open-access publication were covered by a Research Centre of the Helmholtz Association.

Edited by: Kusumita Arora

Reviewed by: Sergey Khomutov and two anonymous referees

\section{References}

Brunke, H.-P., Korte, M., and Widmer-Schidrig, R.: IndCoilsFGE - A software package for merging Fluxgate and Induction Coil data V.1.0, GFZ Data Services, https://doi.org/10.5880/GFZ.2.3.2017.002, 2017.

Korepanov, V. and Rasson, J.: New INTERMAGNET fluxgate magnetometer, publications of the institute of geophysics, Pol. Acad. 75 Sc., 398-406, 2007.

Pedersen, L. W. and Merenyi, L.: The FGE Magnetometer and the INTERMAGNET 1 Second Standard, J. Ind. Geophys. Union, 30-36, 2016.

Ritter, O. and Pulz, E.: Entwicklung einer Kalibriereinrichtung für Induktionsspulenmagnetometer, Scientific Technical Report 01/10 des GeoForschungsZentrum Potsdam, https://doi.org/10.2312/GFZ.b103-0100, 2001.

Schmucker, U. and Weidelt, P.: Electromagnetic Induction in the Earth, Lectures Notes Aarhus, https://doi.org/10.1038/ncomms12883, 1975.

Shprits, Y., Drozdov, A., and Spasojevic, M.: Wave-induced loss of ultra-relativistic electrons in the Van Allen radiation belts, Nature Communications, 2016.

Turbitt, C.: INTERMAGNET Definitive One-Second Data Standard, INTERMAGNET Technical Note, http://www.intermagnet.org/publications/im_tn_06_v1_0.pdf, 2014.

Usanova, M. E., Drozdov, A., Orlova, K., Mann, I. R., Shprits, Y., Robertson, M., Turner, D. L., Milling, D. K., Kale, A., Baker, D. N., Thaller, S. A., Reeves, G. D., Spence, H. E., Kletzing, C., and Wygant, J.: Effect of EMIC waves on relativistic and ultrarelativistic electron populations: Groundbased and Van Allen Probes observations, Geophys. Res. Lett., https://doi.org/10.1002/2013GL059024, 2014. 